\title{
Total cross section model with uncertainty evaluated by KALMAN
}

\author{
Shintaro Hashimoto ${ }^{1, *}$ and Tatsuhiko Sato ${ }^{1,}$ \\ ${ }^{1}$ Japan Atomic Energy Agency
}

\begin{abstract}
We propose a new total cross section model including its uncertainty to estimate systematic uncertainty in particle transport simulations. Medians and widths of parameters in the model, which describe the model uncertainty in the simulation, were determined by using the KALMAN code based on the Bayes' theorem. We implemented the new model in the particle and heavy ion transport code system (PHITS), and then performed shielding calculations with neutron beams. By using analysis of variance (ANOVA), the systematic uncertainty associated with the model uncertainty can be estimated from variance in calculated results.
\end{abstract}

\section{Introduction}

Particle transport simulations based on the Monte Carlo technique have been successfully applied to radiation shielding in accelerator facilities. The reliability of the simulation results can be usually evaluated by statistical uncertainties, which depend on the number of trials. In addition, the results include systematic uncertainties that are caused by unclear physical quantities such as total cross sections and reaction cross sections. Therefore, estimation of the systematic uncertainty is also required to confirm quantitatively the reliability of results.

Recently, Koning and Rochman proposed the Total Monte Carlo method[1] to estimate the systematic uncertainty. In the method, many nuclear data libraries are developed by a nuclear reaction model TALYS[2] with varying its internal parameters. Thereafter, transport simulations are performed along with the developed libraries. The systematic uncertainty can be estimated from variance in results obtained by the simulation. Note that it is difficult to apply this method to analysis of shielding calculation for high-energy accelerators because nuclear reaction models instead of nuclear data libraries are generally employed in the simulation.

In this study, we proposed a new total cross section model implemented in the Particle and Heavy Ion Transport code System (PHITS)[3] to estimate the systematic uncertainty caused by total cross sections of neutrons in the shielding calculation. We evaluated uncertainties of internal parameters included in the model by the code KALMAN[4], which is Bayesian code based on the least squares technique, comparing with experimental data of the total cross section for several targets between $\mathrm{C}$ and $\mathrm{Pb}$. In our method, particle transport simulations are performed varying the internal parameters to obtain the variance in calculated results, and then systematic uncertainties are estimated from the variance based on analysis of variance (ANOVA) [5, 6]. Computational procedures used

*e-mail: hashimoto.shintaro@jaea.go.jp in this study are presented in Sec. 2 and calculation results and discussion are shown in Sec. 3. Section 4 presents our conclusions and future works.

\section{Computational procedures}

\subsection{The KALMAN code}

The KALMAN code[4] based on Bayes' theorem has been used to evaluate covariance data of nuclear data. In this code, the model calculation $f(\mathbf{x})$ with parameter vector $\mathbf{x}$ is assumed by using a first-order Taylor expansion as follows:

$$
f(\mathbf{x})=f\left(\mathbf{x}_{\text {pri }}\right)+\mathbf{C}\left(\mathbf{x}-\mathbf{x}_{\text {pri }}\right),
$$

where $\mathbf{x}_{\text {pri }}$ is the prior parameter vector, and $\mathbf{C}$ is the sensitivity matrix. Elements of the sensitivity matrix are defined as,

$$
C_{a b}=\left(\frac{\partial f_{a}(\mathbf{x})}{\partial x_{b}}\right)_{\mathbf{x}=\mathbf{x}_{\text {pri }}},
$$

where $f_{a}$ is a calculated cross section of the model $f(\mathbf{x})$ at the neutron energy $E_{a}$, and $x_{b}$ is the $b$-th parameter of $\mathbf{x}$. Based on the above assumption and Bayes' theorem, the posterior parameter vector $\mathbf{x}_{\mathrm{pos}}$ is given as,

$$
\begin{aligned}
\mathbf{x}_{\mathrm{pos}} & =\mathbf{x}_{\mathrm{pri}}+\mathbf{P C}^{t} \mathbf{V}^{-1}\left(\mathbf{y}-f\left(\mathbf{x}_{\mathrm{pri}}\right)\right) \\
& =\mathbf{x}_{\mathrm{pri}}+\mathbf{X} \mathbf{C}^{t}\left(\mathbf{C X C} \mathbf{C}^{t}+\mathbf{V}\right)^{-1}\left(\mathbf{y}-f\left(\mathbf{x}_{\mathrm{pri}}\right)\right)
\end{aligned}
$$

and the covariance matrix of the posterior model parameter $\mathbf{P}$ is given by,

$$
\begin{aligned}
\mathbf{P} & =\left(\mathbf{X}^{-1}+\mathbf{C}^{t} \mathbf{V}^{-1} \mathbf{C}\right)^{-1} \\
& =\mathbf{X}-\mathbf{X C}^{t}\left(\mathbf{C X C} \mathbf{C}^{t}+\mathbf{V}\right)^{-1} \mathbf{C X},
\end{aligned}
$$

where $\mathbf{C}^{t}$ is the transpose matrix of $\mathbf{C}, \mathbf{V}$ is the covariance matrix of experimental data, $\mathbf{X}$ is the covariance matrix of 
the prior model parameter and $\mathbf{y}$ is the vector of experimental data.

The KALMAN code uses $\mathbf{x}_{\text {pri }}, \mathbf{X}, \mathbf{C}, \mathbf{y}$, and $\mathbf{V}$ as input data, and then outputs $\mathbf{x}_{\mathrm{pos}}$ and $\mathbf{P}$. In the present study, we used $\mathbf{x}_{\text {pos }}$ and diagonal elements of $\mathbf{P}$, respectively, as medians and widths of the model parameters in the new total cross section model.

\subsection{Total cross section model}

We developed the new total cross section model for neutron-induced reactions based on Pearlstein's formulae[7]. The formulae of the total and non-elastic cross sections at the neutron energy $E$ are defined as follows:

$$
\begin{aligned}
\sigma_{\text {tot }}= & \sigma_{\text {ne }}\left(1+k_{4}\right) \\
& +k_{1} A^{1 / 3} \sum_{h=1}^{2} \exp \left[-\left\{k_{2} \log \left(E_{\mathrm{P} h} / E\right)\right\}^{2}\right],
\end{aligned}
$$

and

$$
\begin{aligned}
\sigma_{\mathrm{ne}}= & p_{1} A^{p_{2}}\left[1-p_{3} \exp \left(-E / p_{4}\right) \sin \left(p_{5} E^{-p_{6}}\right)\right], \\
& \times\left[1+p_{7} \sin \left(p_{8}-p_{9} \log A\right)\right]
\end{aligned}
$$

respectively, where $E_{\mathrm{P} 1}=k_{3} A^{1 / 3}$ and $E_{\mathrm{P} 2}=E_{\mathrm{P} 1}-$ $82(A / 238)^{1 / 3}$. Parameters $k_{m}(m=1, \cdots, 4)$ are given as,

$$
\begin{aligned}
k_{m}= & \exp \left[\left(\log q_{m 1}\right)+\left(\log q_{m 2}\right)(\log A)\right. \\
& \left.+\left(\log q_{m 3}\right)(\log A)^{2}\right] .
\end{aligned}
$$

The total number of parameters is $21, p_{l}(l=1, \cdots, 9)$ and $q_{m n}(m=1, \cdots, 4 ; n=1, \cdots, 3)$.

Medians and widths of the 21 parameters shown in Table 1 were determined by using the KALMAN code and available experimental data of total cross sections for ${ }^{\text {nat }} \mathrm{C}$, ${ }^{27} \mathrm{Al}$, ${ }^{\text {nat }} \mathrm{Fe}$, ${ }^{\text {nat }} \mathrm{Sn}$, and ${ }^{\text {nat }} \mathrm{Pb}$ targets[8]. By changing values of the 21 parameters randomly and independently, the uncertainty of the total cross section model is expressed.

Figure 1 shows neutron total cross sections for the selected targets. Closed circles represent experimental data obtained from the EXFOR database[8]. Calculated total cross sections of the new model using the medians of the 21 parameters are denoted by the solid line, and model uncertainties estimated by the calculation with varying the parameters from a Gaussian distribution with the widths are represented by the error bars. The agreements between the model uncertainties and dispersions of experimental data are generally satisfied, though our model tends to give smaller and larger uncertainties for lighter and heavier targets, respectively.

\subsection{PHITS particle transport simulation}

We performed two shielding calculations with neutron beams of $100 \mathrm{MeV}$ by using PHITS to confirm the validity of the new total cross section model for estimation of the systematic uncertainty in Monte Carlo simulations. The shielding material was assumed as a cylinder made of concrete or lead. As calculated results, effective doses in the material were obtained applying the dose conversion
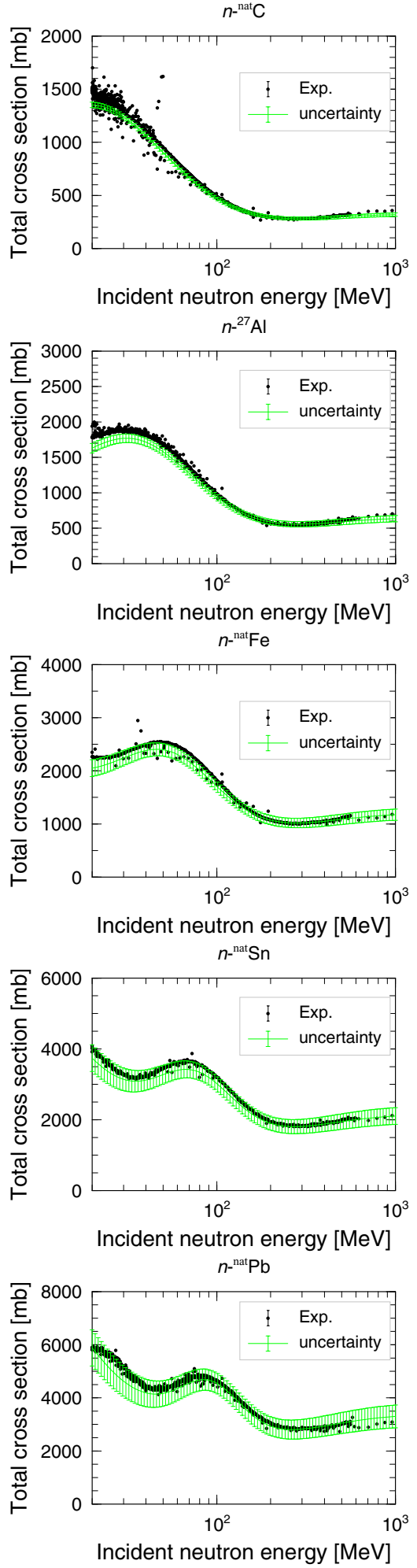

Figure 1. Total cross sections of neutron-induced reaction for ${ }^{\text {nat }} \mathrm{C},{ }^{27} \mathrm{Al}$, ${ }^{\text {nat }} \mathrm{Fe}$, ${ }^{\text {nat }} \mathrm{Sn}$, and ${ }^{\text {nat }} \mathrm{Pb}$ targets. Solid lines and error bars denote the total cross sections and their uncertainty calculated by the new model, respectively. Available experimental data obtained from EXFOR[8] are also shown.

coefficients[9]. When concrete was assumed as the shielding material, we set the cylinder with a radius of $10 \mathrm{~cm}$ and thickness of $100 \mathrm{~cm}$. Information on atomic fraction and density of the concrete was taken from ANL-5800[10]. On 
Table 1. Medians and widths of the 21 model parameters, $p_{l}(l=1, \cdots, 9)$ and $q_{m n}(m=1, \cdots, 4 ; n=1, \cdots, 3)$.

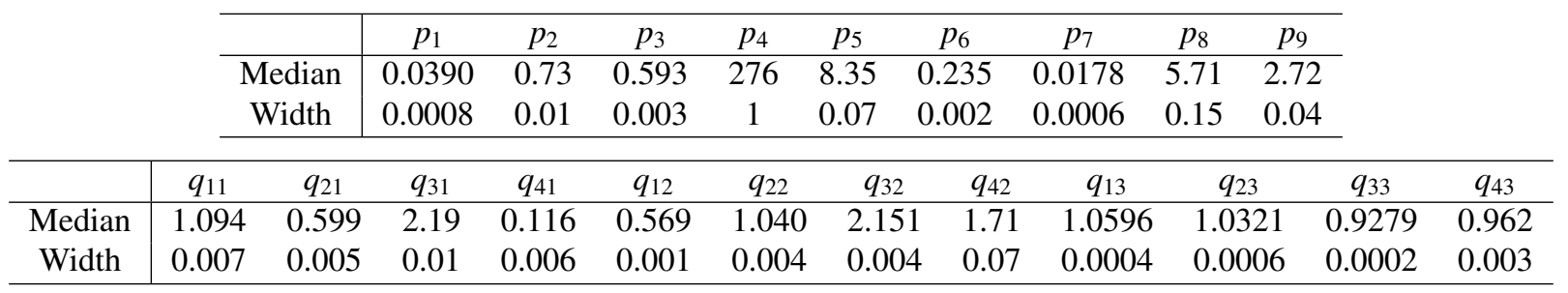

the other hand, we set the cylinder with a radius of $10 \mathrm{~cm}$ and thickness of $10 \mathrm{~cm}$, when the material was assumed to be lead. The axis of the neutron beam was set along the central axis of the cylinder in the two cases.

In this study, we implemented the new model in PHITS, and then applied it to shielding calculations with neutron beams. The PHITS calculation was performed with the default setting for describing nuclear reaction processes, except for the total cross sections of neutrons with energies higher than $20 \mathrm{MeV}$. We used the fourth version of the Japanese Evaluated Nuclear Data Library (JENDL4.0)[11] for neutron energies of below $20 \mathrm{MeV}$. The Liège intranuclear cascade model version 4.6 (INCL4.6)[12] was used to describe the inelastic scattering processes of the neutrons.

Systematic uncertainties associated with the uncertainty of the total cross section model were estimated from variance in calculated results by performing ANOVA (analysis of variance)[5, 6]. This method gives systematic and statistical uncertainties, $u_{\text {syst }}$ and $u_{\text {stat }}$, by calculating

$$
\begin{aligned}
& \frac{1}{J-1} \sum_{j}^{J} \sum_{i}^{I}\left(\bar{x}_{j}-\bar{x}\right)^{2}=I u_{\text {syst }}^{2}+u_{\text {stat }}^{2}, \\
& \frac{1}{J(I-1)} \sum_{j}^{J} \sum_{i}^{I}\left(x_{i j}-\bar{x}_{j}\right)^{2}=u_{\text {stat }}^{2},
\end{aligned}
$$

where $x_{i j}$ is a calculated result in a trial $i$ with a condition $j$, which indicates using one set of the 21 parameters of the new total cross section model. $I$ and $J$ are the total number of $i$ and $j$, respectively. $\bar{x}_{j}$ is the mean value of the calculated results under the condition $j$, and $\bar{x}$ is the mean value of all results.

\section{Results and Discussion}

Figure 2-(a) shows depth distribution of effective doses in the concrete material. Mean values obtained by the PHITS calculation with $J=10^{3}$ and $I=10^{6}$ are denoted by the solid line, and the systematic uncertainties are represented by error bars. The numbers $J$ and $I$ are sufficiently large to obtain the converged results, and the statistical uncertainties are negligible in the results. The uncertainties become gradually larger as the depth increases, because the doses at deeper location are more sensitive to the value of the cross section due to multiple scattering. Relative values of the uncertainties to the mean values are shown in Fig. 2-(b). It is found that there is an almost linear dependence of the relative uncertainty on the depth.
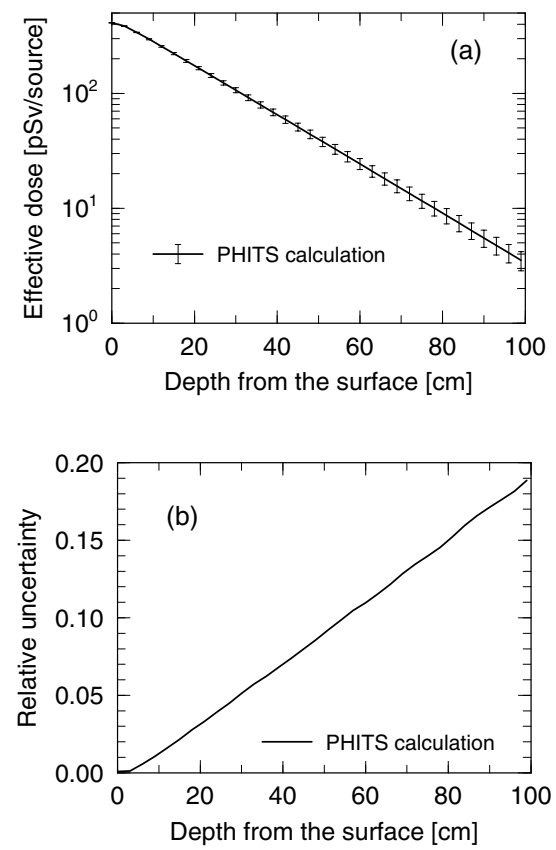

Figure 2. (a) Effective doses in concrete shielding material induced by $100 \mathrm{MeV}$ neutrons. The solid line and error bars denote the mean values and systematic uncertainties calculated by PHITS. (b) Relative values of the uncertainties to the mean values.

The depth distribution of the effective doses in the lead material is shown in Fig. 3-(a). The mean values and systematic uncertainties calculated by PHITS with $J=10^{3}$ and $I=10^{6}$ are represented by solid lines and error bars, respectively. Figure 3-(b) shows the depth distribution of the relative uncertainties. The large uncertainties are found in the surface (build-up) region and deep region. On the other hand, the uncertainties in the intermediate region around $4 \mathrm{~cm}$ are very small. This trend can be understood by considering the influence of the variation of the total cross sections, as discussed in the previous study[6]. When the cross sections are increased, the effective dose is suppressed in the deep region, and that in the surface region is enhanced by the scattered neutrons. On the other hand, decreasing the cross sections causes the opposite shift. Therefore, the systematic uncertainties around $4 \mathrm{~cm}$ become small owing to the mechanism.

\section{Summary}

In this work, we presented a new total cross section model of neutrons with its uncertainty. Medians and widths of the 

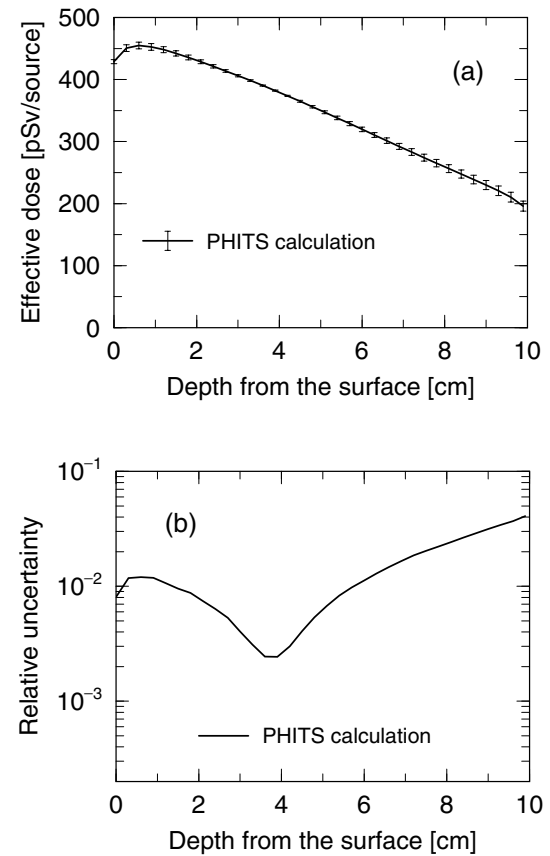

Figure 3. (a) Effective doses in lead shielding material bombarded with $100 \mathrm{MeV}$ neutron beam. The mean values and systematic uncertainties are denoted by the solid line and error bars. (b) Relative uncertainties of the calculated results.

model parameters, which correspond to the uncertainty of the model, were determined by the KALMAN code. The model was implemented in PHITS and then applied to analyses of two neutron-shielding calculations. Effective doses in the shielding material were calculated by PHITS simulation varying the values of the model parameters. By using ANOVA, we estimated systematic uncertainties caused by the uncertainty of the total cross section model in the simulation from variance of calculated results.

We plan to improve this model to revise the underestimation in the reactions of light nuclei and consider reaction cross sections by adding several model parameters.
The uncertainties of the nuclear reaction models such as intranuclear cascade and evaporation models are also desirable to be considered in future, which requires additional studies.

\section{Acknowledgement}

This work was partially supported by JSPS KAKENHI Grant Numbre 17K14917.

\section{References}

[1] A.J. Koning and D. Rochman, Ann. Nucl. Energy 35, 2024-2030 (2008).

[2] A.J. Koning, S. Hilaire, M.C. Duijvestijn, Proc. Int. Conf. on Nuclear Data for Science and Technology ND2007; 2007 May 22-27; Nice (France). p.211-214.

[3] Sato T et al., J. Nucl. Sci. Technol. 55, 684-690 (2018).

[4] T. Kawano and K. Shibata, JAERI-Data/Code 97-037 (1997) [in Japanese].

[5] Casella G., Statistical design (Springer, Berlin, 2008). p.2-10.

[6] Hashimoto S and Sato T, J. Nucl. Sci. Technol. 56, 345-354 (2019).

[7] S. Pearlstein, Astrophys. J., 346, 1049 (1989)

[8] IAEA-EXFOR experimental nuclear reaction data base, http://www.nds.iaea.org/exfor

[9] Y. Sakamoto et al., Tokai, Japan: Japan Atomic Energy Research Institute; 2003. (JAERI-1345). [in Japanese].

[10] Argonne National Laboratory. Reactor Physics Constants. USA: United States Atomic Energy Commission; 1963 Jul, ANL-5800 (2nd Ed.). p.660.

[11] K. Shibata et al., J. Nucl. Sci. Technol. 48, 1-30 (2011).

[12] A. Boudard et al., Phys. Rev. C 87, 014606 (2013). 